\title{
Measuring the Performance Characteristics of a Motorcycle
}

Adam Hamberger, Milan Daňa

Regional Technological Institute, University of West Bohemia - Faculty of Mechanical Engineering, Univerzitní 8, Pilsen 306 14, Czech Republic. E-mail: adam26@students.zcu.cz

This work deals with an experiment, whose output is the comparison of power characteristics which were measured in three ways. The first way used a commercially manufactured dynamometer. For the second measurement, a special dynamometer with our own computing system and a sensor within this project was created. The last way of measuring the performance characteristics was done without a dynamometer. The measurement works on the principle of acceleration the spinning of a flywheel. Due to this, the measuring is called an acceleration test. The basic principles are described before the experiment in order to grasp the characteristics. All explanations are based on schemes and easy mathematical and differential formulas to describe the construction of the dynamometer and the principle of its functions from the engine to the computer. The relations between published and unpublished quantities defining engine dynamics are explained here. In the end, this work points to possible and intended measuring failures which are an infamous practice at many measuring stations.

Keywords: Dynamometer, power characteristics, driving forces, torque, performance.

\section{Introduction}

All vehicle engines overcome resistances on the road.

$$
F_{r e s}(v)=c_{2} \cdot v^{2}+c_{1} \cdot v+\left(c_{v a l}+\sin (\phi)\right) \cdot m \cdot g \quad[N]
$$

where:

$\mathrm{v}$... velocity $[\mathrm{m} / \mathrm{s}]$,

$\mathrm{c}_{1}, \mathrm{c}_{2}, \mathrm{c}_{\text {roll }} \ldots$ coefficients [-],

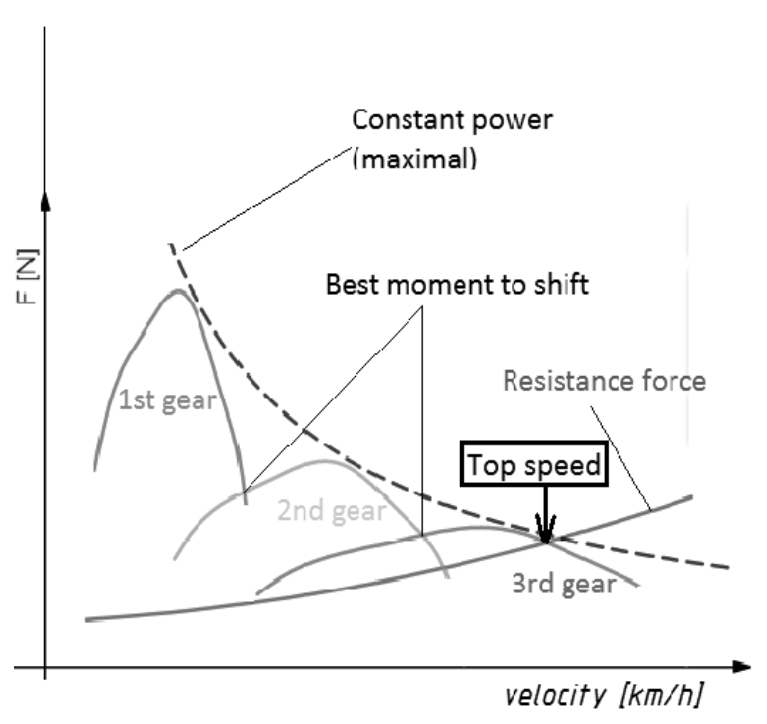

These forces are divided into two basic sorts. The first one is dependent on velocity and it can be written as: $\varphi$... climb angle [rad],

$\mathrm{m}$... full weight $[\mathrm{kg}]$,

$\mathrm{g}$... gravity acceleration $\left[\mathrm{m} / \mathrm{s}^{2}\right]$.

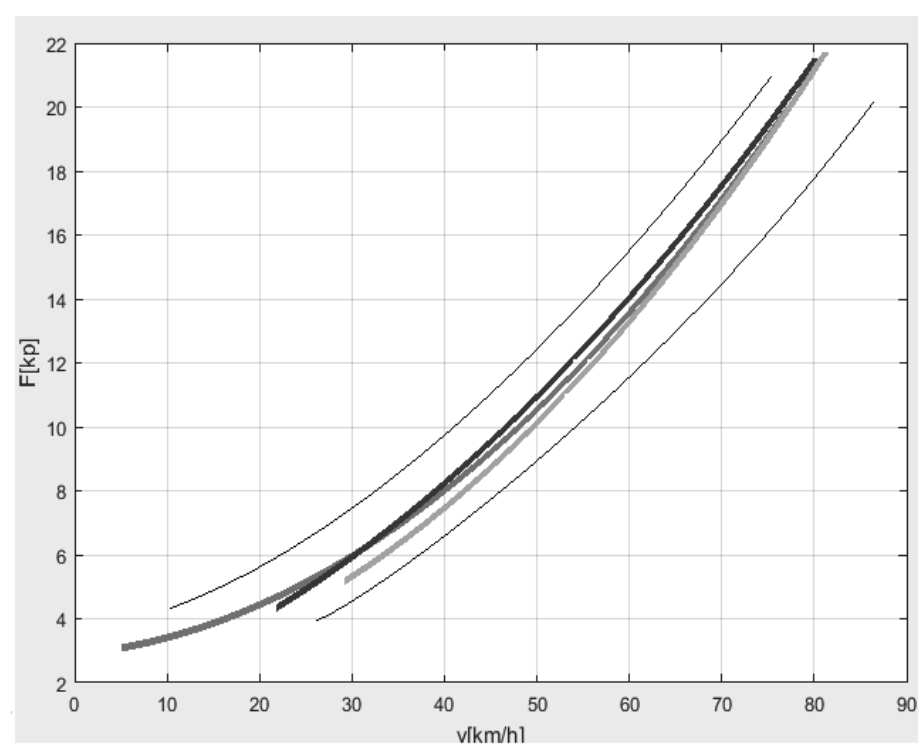

Fig. 1 Drive force diagram and experimentally determined resistance forces on the road

Most technical literature does not take the linear element into consideration. But this is a mistake, because as this experiment proved, it is not possible to approximate the resistance equation without the linear element. This element takes into consideration viscous friction, i.e. air friction and friction in the chain and other components. The second sort of forces are inertial effects, and they are dependent on acceleration.[2],[8],[12]

Before defining the known quantities, it is necessary to explain what characterizes vehicle dynamics. Based on
Newton's second law it is possible to create the equation of vehicle acceleration:

$$
a(t)=\frac{F_{d}(v)-F_{r e s}(v)}{m_{\text {red }}} \quad\left[\frac{m}{s^{2}}\right]
$$

where:

$\mathrm{t}$... time $[\mathrm{s}]$,

$\mathrm{F}_{\mathrm{d}} \ldots$ drive force $[\mathrm{N}]$,

$\mathrm{F}_{\text {res }}$... resistance force $[\mathrm{N}]$,

$\mathrm{m}_{\text {red }} .$. reduced mass $[\mathrm{kg}]$, 
Because the main purpose is to analyse the engine characteristics, it is necessary to focus on the drive force. The meaning of reduced mass will be explained below.

\section{Describing the characteristics}

Here are explained the basic principles of the performance characteristics. Then the method to determine the characteristics and resistance forces are explained in order to understand the experiment in section 3.

\subsection{Drive force}

Drive force is the tangential force between the tyre and the road during acceleration. This equation gives the relation between drive force and engine torque.

$$
F_{d}(v)=\frac{M_{t}(n) \cdot i_{\text {total }}}{r_{\text {tyre }}} \quad[N],
$$

where:

$\mathrm{M}_{\mathrm{t}} \ldots$ crankshaft torque $[\mathrm{Nm}]$, n ... crankshaft revolutions [RPM], $i_{\text {total }} . .$. total gear ratio of vehicle $[-]$, $\mathrm{r}_{\text {tyre }} \ldots$ drive tyre radius $[\mathrm{m}]$.

Every gear has its gear ratio with continuous drive force. As you can see in Fig.1 and Fig.2 the force curves and torque curves are similar but deformed in the direction of hyperbolas. Both torque curves can achieve the same force curve by means of another gear ratio. Despite this, most people would say that the engine with the red curve is stronger because its torque is higher. A typical output of a dynamometer is a graph with a power curve and a torque curve. Therefore the best way to determine vehicle power is using a drive force diagram (for example when tuning, etc.).

\subsection{Torque vs. power}

Because it is necessary to know all the gear ratios to create a drive force diagram, it is not common to publish it. The torque curve has the same course as the drive force curve. This means that the pull forward is characterized by the course of the torque, not by the course of the power. This means that the torque diagram is graphical interpretation of vehicle acceleration. Despite this, the torque diagram is ignored in most cases. The most frequent question after measuring is, "How much horsepower does it have?" This is very important question but without the torque curve it is not so substantial. The most important fact is that the maximum torque value is totally unnecessary. The mathematical relation between torque $\left(M_{t}\right)$ and power $(P)$ is shown in Fig. 2.

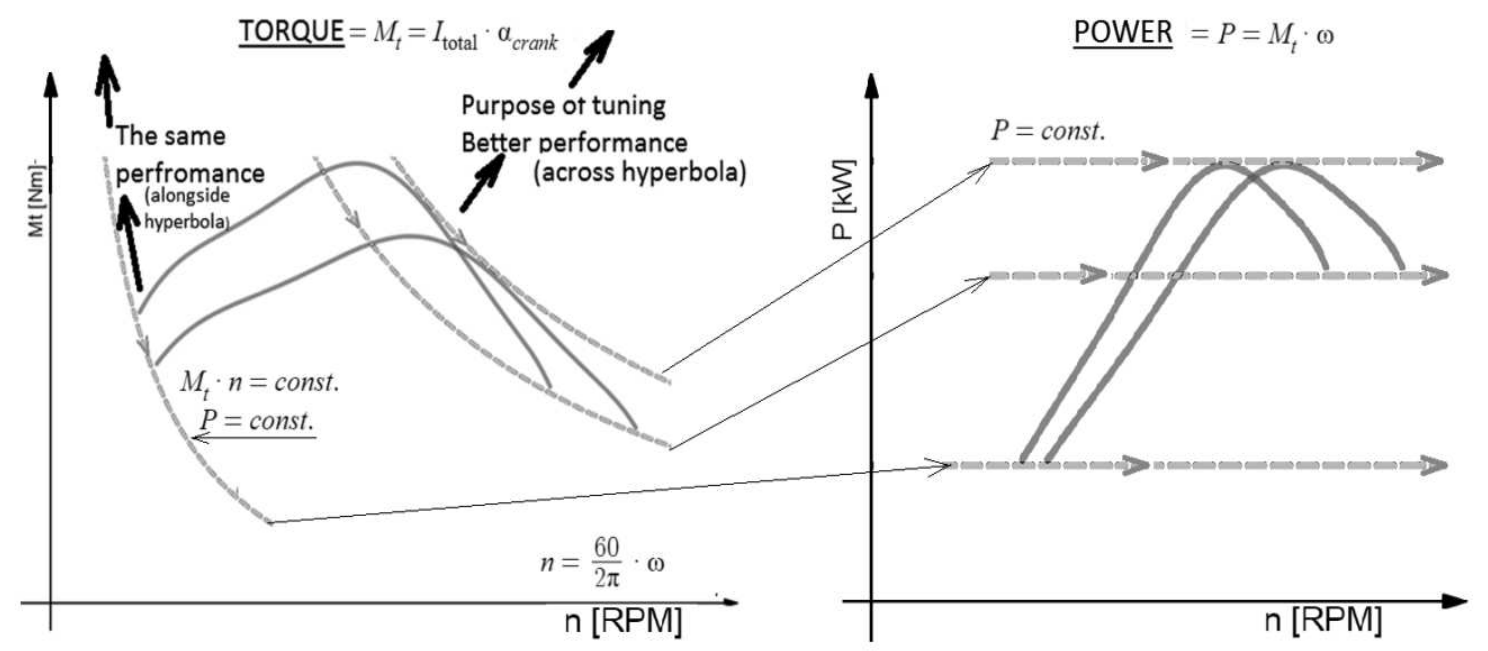

Fig. 2 Two engines with the same performance

\subsection{Principle of measuring}

In most cases, the engine is measured using a dynamometer (abbreviated to DYNO). The main component of the dynamometer is the flywheel. The flywheel is rotated by the engine power (mostly via the wheel). The speed of the flywheel is monitored during measuring. Measuring is composed of two phases - acceleration and deceleration tests. During the acceleration test, the flywheel is rotated with the engine torque. The operator opens the throttle at minimum engine speed. The flywheel is rotated to maximum engine speed and it is monitored using a revolution sensor. It is the end of acceleration test and then the measured data are analysed in a computer.

The flywheel has a scanned point (for example a magnet) and the frame has a sensor (Hall sensor). The signal from the sensor is transferred to the computer. Every revolution of the flywheel makes a pulse and the time between the impulses is marked as $d t$. The full angle of every revolution $\left(360^{\circ}=2 \pi \mathrm{rad}\right)$ is marked as $d \varphi$. Now it is possible to create the angular velocity for all revolutions, for example 100 values of the scanned flywheel. These numbers are approximated by the polynomial function of degree 15 because it is easy to derivate. This polynomial function $\omega(t)$ is again derived in order to ascertain angular acceleration $\alpha$. The best way to process the calculated data is to create a matrix with rows for every revolution. Then it is possible to change from time variable to RPM variable and to multiply by inertia moment in order to ascertain torque, etc. All these calculations are carried out using the reduced inertia mass method, which 
is described in [6]. Aforesaid part of measuring is called acceleration test.

The second part of measuring is the deceleration test. The purpose of this test is to determine the resistance forces. After the acceleration test at the highest speed, the engine is separated by shifting into neutral. All masses, which are separated from the engine, turn by themselves until they stop. This run is monitored as well as acceleration. Both parts of the measurements are shown on the Fig. 3.

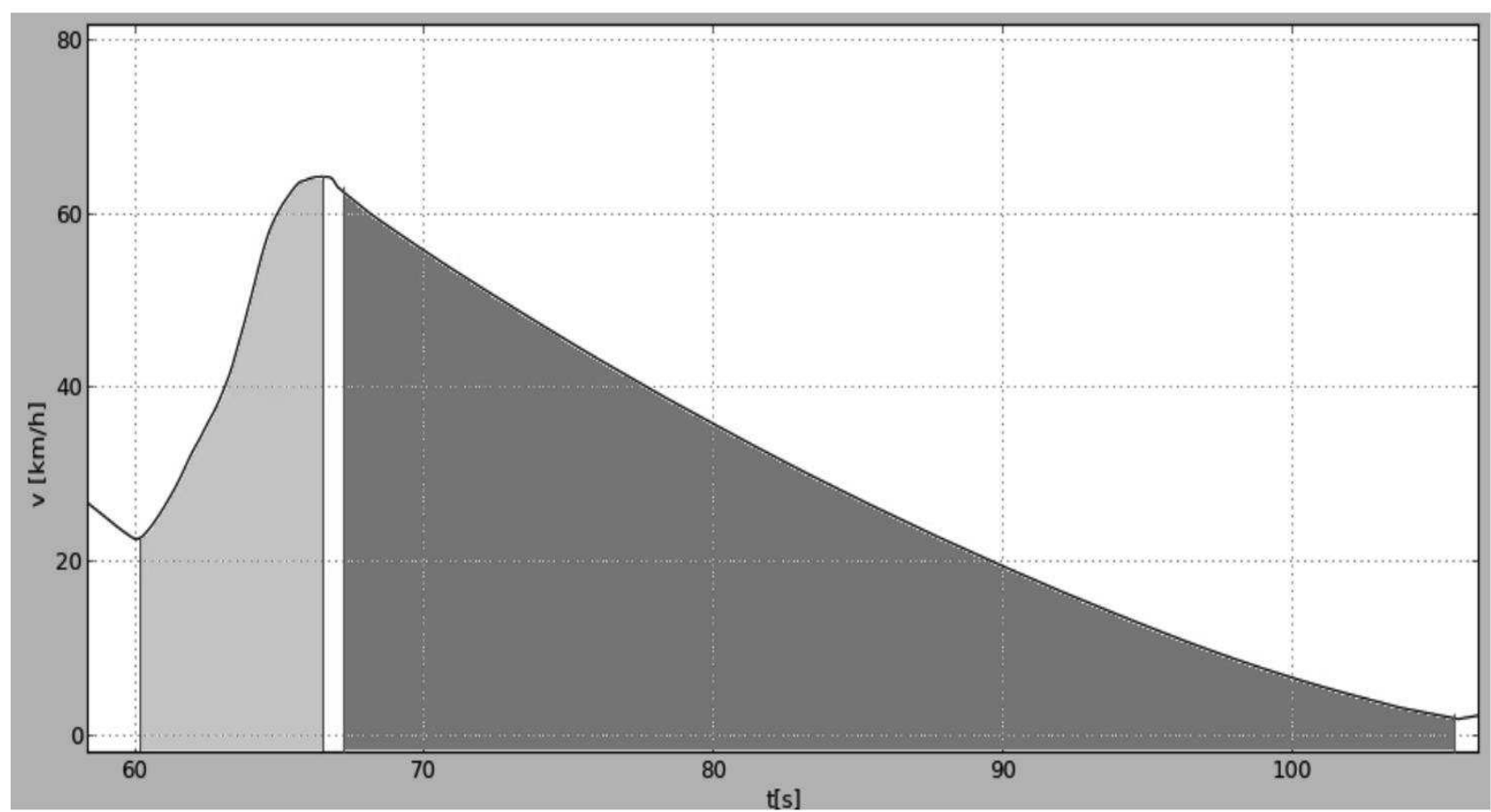

Fig. 3 Velocity by measuring

The whole data processing is done in the same way. So the measured torque is added to the values which were determined in the acceleration test. This sum total can be written as the engine torque and used to calculate other values (drive force, power). This equation explains the simplified way to determine the reduced moment of inertia when the crankshaft and dyno-flywheel spin:

$$
I_{\text {redtocrank }}=I_{\text {crank }}+\frac{I_{f}}{i_{\text {total }}^{2}} \quad\left[\mathrm{~kg} \cdot \mathrm{m}^{2}\right],
$$

where:

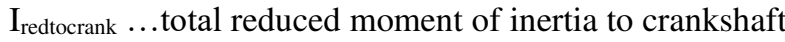
$\left[\mathrm{kg} \cdot \mathrm{m}^{2}\right]$,

$\mathrm{I}_{\mathrm{f}} .$. moment of inertia of flywheel $\left[\mathrm{kg} \cdot \mathrm{m}^{2}\right]$,

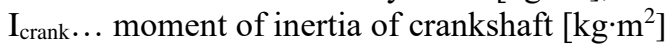

$\mathrm{I}_{\text {total }}$.. total gear ratio between flywheel and crankshaft $\left[\mathrm{kg} \cdot \mathrm{m}^{2}\right]$

This value of total moment is multiplied by the angular acceleration of the crankshaft in order to ascertain the crankshaft torque for the acceleration itself. As already mentioned, for the purpose of ascertaining total torque, the torque of passive resistance must be added. The torque of passive resistance is scalar product of angular acceleration when deceleration test and of inertia moment of fly- wheel (without crankshaft), because the crankshaft is separated from the flywheel during the deceleration test. This is a simplified way to describe this process. In order to achieve the most accurate results, the moment of inertia is calculated for all moving parts using a CAD system. Inertia calculated in this way can be approximately 80$98 \%$ of the inertia mass of all the moving parts.

\section{Experiment}

This experiment deals with determining the performance characteristics of the same motorcycle in three ways. The first measurement is carried out on a commercially available dynamometer with its own software and sensors. We made our own dynamometer for the second measurement and its software was made using a graphical user interface. The third measuring method does not need a dynamometer. This method is carried out on the road only by means of the system from the second measurement method in order to substitute the inertia of the dynoflywheel for the mass of the motorcycle and rider. The purpose of this experiment is to verify all the mentioned principles and to compare the results of all three ways of measuring. It was necessary to ascertain the inertia of all the moving parts. This was done using CAD models which were created using reverse engineering of used motorcycle components and a dyno-flywheel. 

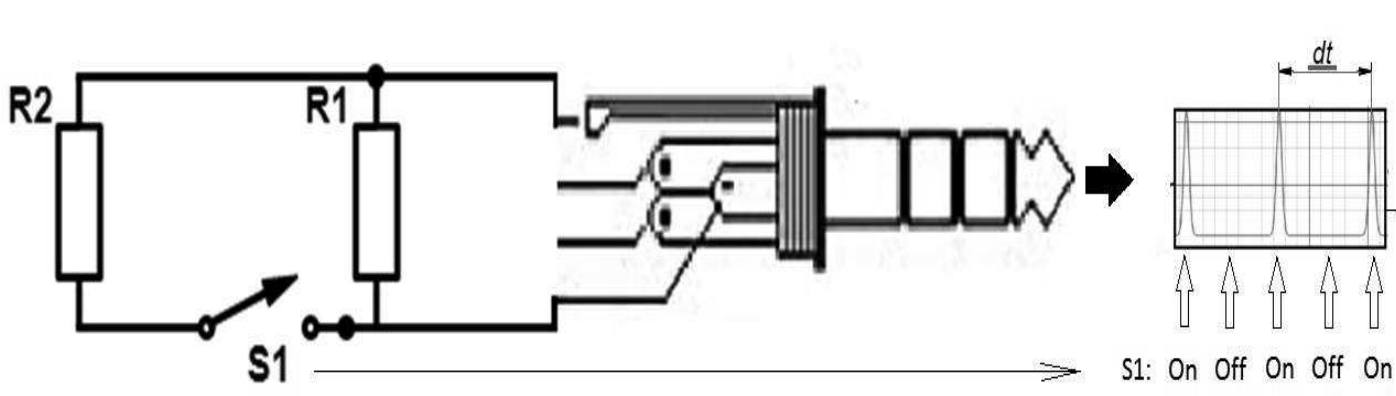

$$
\begin{aligned}
& \omega=\frac{d \varphi}{d t} \\
& \alpha=\frac{d \omega}{d t} \\
& M_{t}=I \cdot \alpha \\
& P=M_{t} \cdot \frac{2 \pi n}{60}
\end{aligned}
$$

Fig. 4 Diagram of scanning sensor

In Fig.4 you can see the function of the scanning device. Due to resistor R1, PC identifies connected device to the soundcard as a microphone. Then the soundcard provides current through this device as well as through the microphone. When the magnet on the scanned wheel goes past the magnetic switch, the device has smaller impedance because the current from the soundcard can go through both parallel resistors. This means that the soundcard detects more signal and it increases the amplitude in the sound recording. When you listen to this recording, you hear a sequence of clicks. Our dynosoftware records this sequence and reads the time intervals (as already mentioned $d t$ ) between the clicks when turning and it calculates other variables from them.

\subsection{Our dynamometer vs. commercial dynamometer}

The commercial dynamometer was hired from a motorcycle service, which engages in measuring motorcycle performance. This dyno was used for measuring the same motorcycle as well as our dynamometer. The commercial dynamometer differs from our version in the hardware and software, because the commercial one uses transmission over the tyre and ours uses the chain transmission to spin the dyno-flywheel. The software is also different, because ours was created without any inspiration from other software, but only on the basis of the physical principles described above. After setting up the required parameters and the acceleration and deceleration tests, the torque and RPM values of both measurements were exported (circa 150 values).The curves of both value lists were created and compared in one graph (Fig.6). About $0.5 \mathrm{Nm}$ difference was caused by an incorrect moment of inertia value, because the dyno manufacturer registered a default value only on the basis of the dyno-flywheel in the software. The correct value must take into account the influence of all moving parts (rear wheel, chain transmission, etc.) and then it is circa $10 \%$ higher.
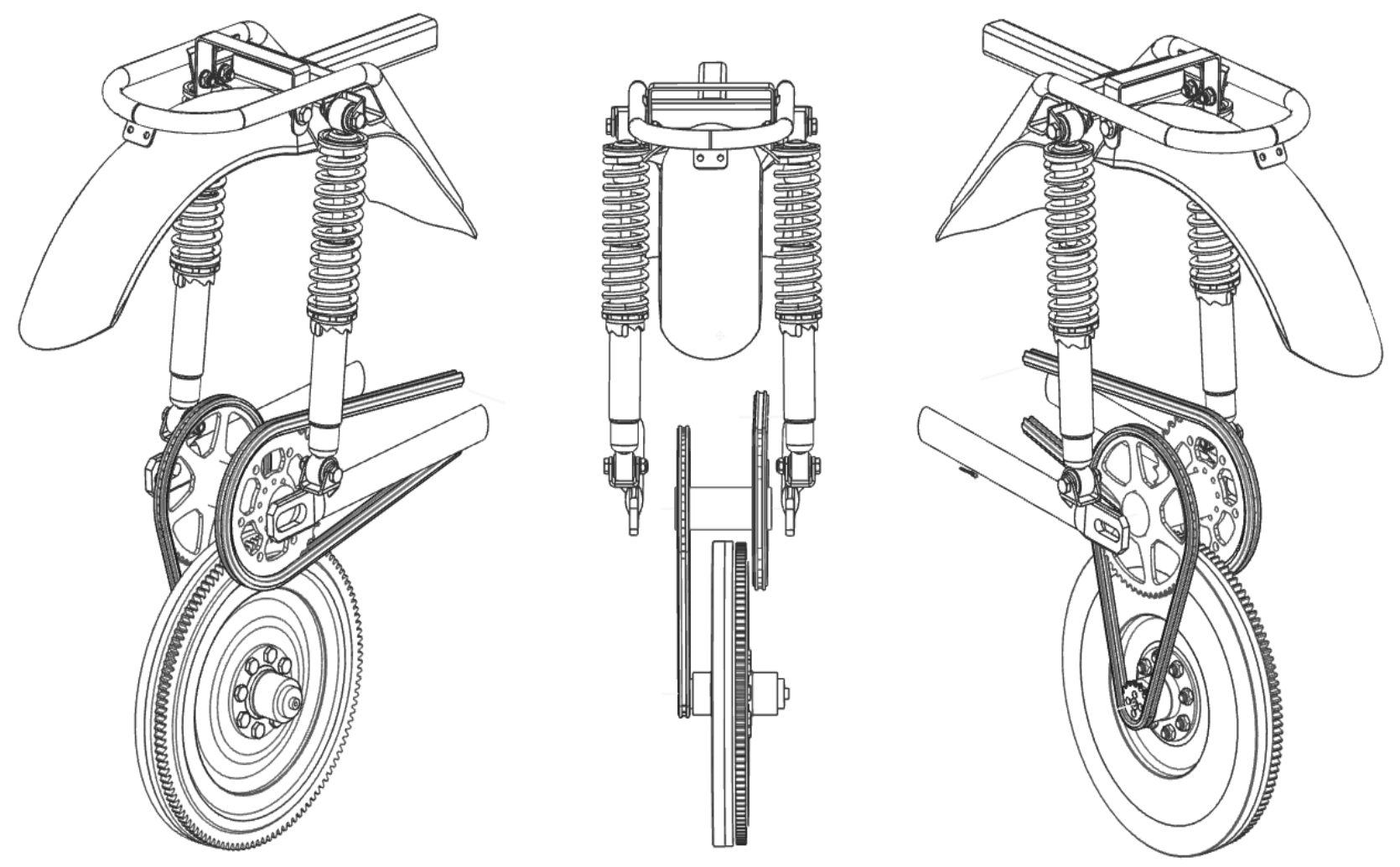

Fig. 5 Flywheel drive in our dynamometer 
This difference is reflected in the torque curve twice, because the software calculated using lower inertia resistance for the acceleration test and with lower passive resistance by for the deceleration test. The second difference between the curves is the smoothness of the blue curve. There are three possible reasons. The first one is friction of the gear over the tyre, because it could absorb sudden alterations of drive force. The second reason could be vibrations of the sensor and changing of the distance between the sensor and the magnet. The last reason could be a part of the computing system. After many tests and changes to the software and hardware, the right reason was found. The commercial software uses the least squares method for approximation by polynomial of degree 10. Our software uses the same method but with a polynomial of degree 13. A polynomial function of higher degree can be more accurate, but it can be too uneven, because the measured RPM values are dispersed due to the tolerance of the measured time intervals $d t$. This tolerance can be reduced with a more rigid sensor holder. After increasing the holder rigidity, editing the polynomial degree and moment of inertia, both curves are almost identical within engine temperature tolerance, etc.

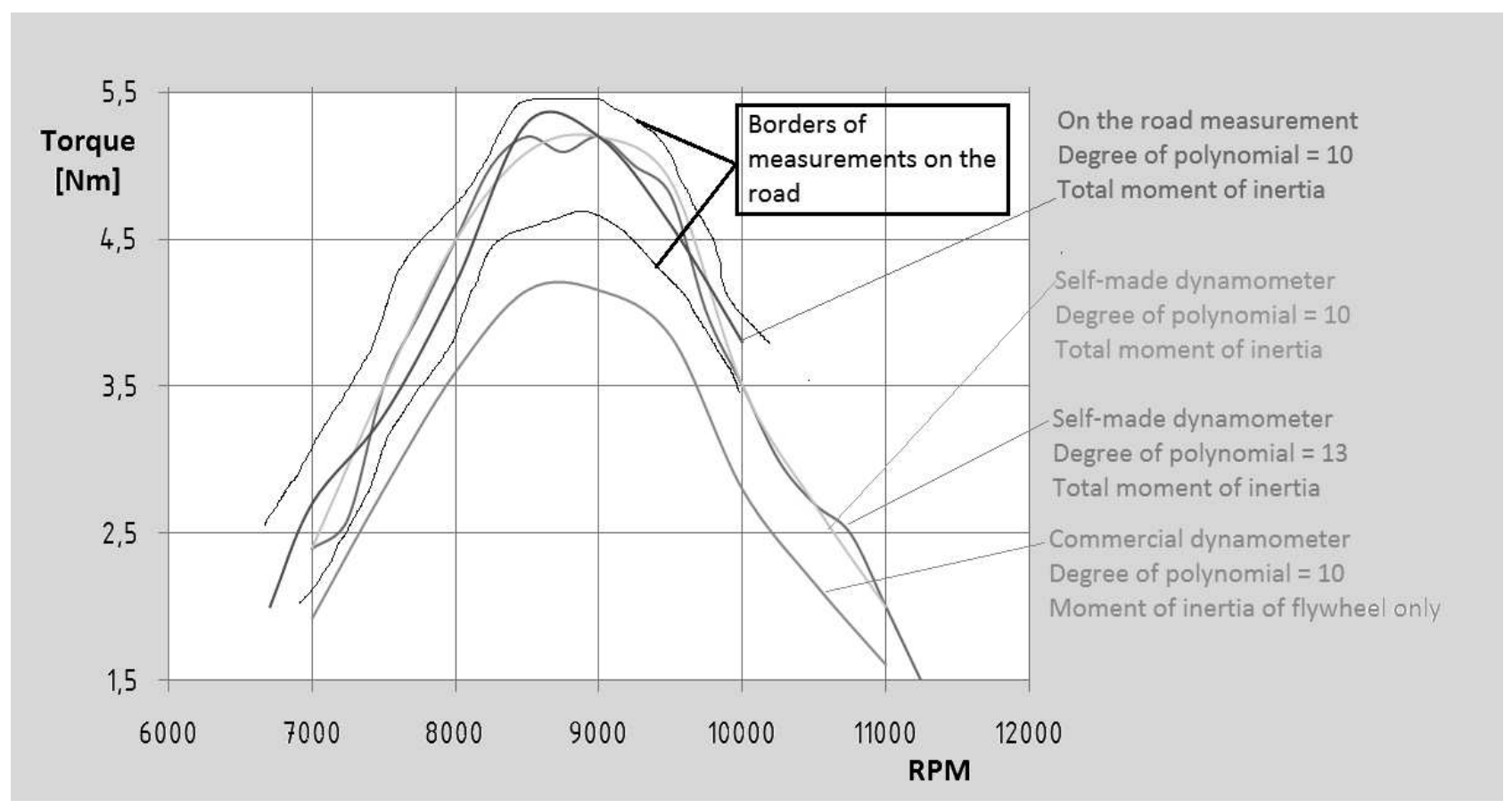

Fig. 6 Comparison of results

\subsection{Measuring on the road vs. our own dynamometer}

The measuring device (Fig.4) was installed on the front motorcycle fork. Both parts of the measuring were carried out on a straight road in one direction. The inertia mass of the rider and the motorcycle substituted for the inertia effect of the dyno-flywheel. The total reduced moment of inertia was calculated from equation 5. There is shown the resistance force function dependent on the velocity approximated by equation 1 in Fig.1. Three colour curves represent the average of 10 measurements, their extreme situations are represented by black curves. The average curve from the acceleration tests was chosen from 10 measurements too. Due to the high variance of the results on the road, it is necessary to make more tests (at least 8 ) because the road conditions are not stable. It is not so good to publish one result on the grounds of one measurement, but if the number of measurements is adequate it is acceptable to publish it. A graphical difference between the dyno and road-curve is visible at high RPM. The curve from measuring on the road does not extend at high RPM, because the air resistance causes braking at high speed and the motorcycle cannot accelerate any more. On the other hand, the engine on the dynamometer can achieve higher revolutions because the resistances are not so intense at high speed.

$$
I_{\text {redtocrank }}=I_{\text {crank }}+\frac{2 \cdot I_{\text {wheel }}+\left(m_{m}+m_{r}\right) \cdot r_{\text {wheel }}^{2}}{i_{\text {total }}^{2}} \quad\left[\mathrm{~kg} \cdot \mathrm{m}^{2}\right] \text {, }
$$

where:

$\mathrm{I}_{\text {wheel }} .$. moment of inertia of wheel $\left[\mathrm{kg} \cdot \mathrm{m}^{2}\right]$

$\mathrm{m}_{\mathrm{m}} \ldots$ mass of motorcycle $[\mathrm{kg}]$

$\mathrm{m}_{\mathrm{r}} \ldots$ mass of rider $[\mathrm{kg}]$

$\mathrm{r}_{\mathrm{wheel}}$... radius of wheel $[\mathrm{m}]$

\section{Conclusion}

This experiment verifies that the described physical principles work in practice by means of measuring in three ways. In order to achieve good reproducibility of measurements, the default inertia value must be edited in 
the commercial software because the inertia of the rear wheel was ignored. The difference in calculating methods (for example polynomial degree) did not have so big influence. The measurement on the road proved that it is acceptable to publish the average result from 10 measurements because the conditions on the road are not stable. This average result is closer to the reality than the result from the commercial dynamometer with default inertia value. In order to research the influence of tuning, it is more necessary to know the curve without accurate power value. It is not necessary to know the accurate inertia value for this, but doing the deceleration test is always very important. The reasons were given for why results from many measuring stations can be different. Results can be published only if the inertia value in the software is true. It is possible only if the values of the main components are known (rear wheel, crankshaft, dyno-flywheel). A common phenomenon at many measuring stations is the correction of the moment of inertia value in order to edit the measured results. It can be done to meet the requirements of a client who wanted to tune his engine from the measuring station (for example). There are cases, where the measurer does not perform the deceleration test and writes a higher inertia value in the software in order to compensate the result. It is very bad approach because the result would be determined on the gearshift of the vehicle even though the engine torque and power are independent of the gear.

\section{Acknowledgement}

This paper is based upon work sponsored by project SGS-2016-005.

\section{References}

[1] PEXA, M. MÜLLER, M. HLOCH, S. (2017) Dynamic measuring of performance parameters for vehicles engines, Measurement. Elsevier.

[2] KLIMENDA, F. SOUKUP, J. (2015). Driving Dynamics of Hybrid Vehicle, Strojirenská technologie, No. 2, ISSN 1211-4162.
[3] PEXA, M. MÜLLER, M. HLOCH, S. (2017) Dynamic measuring of performance parameters for vehicles engines, Measurement. Elsevier.

[4] MARTYR, A.J.M. (2007). Engine testing theory and practice. 3rd ed. Oxford: Butterworth-Heinemann, ISBN 978-075-0684-392

[5] Ding, Z. Q. (2010). Design of motor speed measurement device based on hall effect sensor. Journal of Agricultural Mechanization Research, Tsinghua University, s. 80-83.

[6] STEJSKAL, M. (2012). Increasing single cylinder si two-stroke engine performance, Diploma thesis, Brno University of Technology

[7] BAKHTADZE,A. (2012). About the Methodology of Teaching the Moment of Inertia in Algebra Based Physics Course. Journal of Technical Science and Technologies, ISSN 2298-0032

[8] BETTES, H. HANCOCK,B. (2008). Dyno testing and tuning, Publisher: CarTech, ISBN: 9781934709740

[9] PEXA, M. MÜLlER, M. HLOCH, S. (2017) Dynamic measuring of performance parameters for vehicles engines, Measurement. Elsevier.

[10] MARTYR, A.J. PLINT, M.A. (2007) Chassis or rolling road dynamometers, Engine Testing, Pages 368-394, ISBN: 978-0-7506-8439-2

[11] MERIAM, J.L. KRAIGE, L.G (2013) Engineering mechanics - Dynamics.7th edition.John Wiley $\&$ Sons, Inc.

[12] VALČÁKOVÁ, F. ŠTEFAŇAKOVÁ, V. (2015). Test Stand Dynamics Properties Investigation by Means of Simulation Computations, Manufacturing Technology, Vol. 15, No. 3, ISSN 1213-2489. 